\title{
COMBINATORIAL ASPECTS OF THE ISING MODEL FOR FERROMAGNETISM. II. AN ANALOGUE TO THE WITT IDENTITY
}

\author{
BY S. SHERMAN ${ }^{1}$ \\ Communicated by Daniel Zelinsky, January 15, 1962
}

M. P. Schutzenberger has indicated to the author that the identity [S, p. 208, eqn. 6] used to prove the COROLLARY ON COIN ARRANGEMENTS of [S] is the same as the identity [H, p. 170; W, p. 156] used to establish the Witt formula for the dimension of the linear space of Lie elements of degree $r$ in a free Lie algebra with $k$ generators over a field of characteristic zero. Since almost no American mathematicians read the Journal of Mathematical Physics and no algebraists do, a special case of the main theorem will be presented here without proof and in a form suitable for the algebraist.

First, the Witt identity will be formulated: Let $a_{1}, \cdots, a_{k}$ generate a free semigroup. Consider only circular words of the same length and period [H, p. 170]. Let $M\left(m_{1}, m_{2}, \cdots, m_{k}\right)$ be the number of such circular words with $m_{1}$ occurrences of $a_{1}, m_{2}$ occurrences of $a_{2}$, etc. Let $z_{1}, \cdots, z_{k}$ be commuting indeterminates. Then [H, p. 170; W, p. 156]

$$
1-z_{1}-z_{2}-\cdots-z_{k}=\prod_{m_{1}, \cdots, m_{k} \geq 0}\left(1-z_{1}^{m_{1}} \cdots z_{k}^{m_{k}}\right)^{M\left(m_{1}, \cdots, m_{k}\right)}
$$

From this the Witt formula,

$$
M\left(m_{1}, \cdots, m_{k}\right)=m^{-1} \sum_{d \mid m_{1}, \cdots, m_{k}} \mu_{(d)} \frac{\left(\frac{m}{d}\right) !}{\left(\frac{m_{1}}{d}\right) ! \cdots\left(\frac{m_{k}}{d}\right) !},
$$

where $m=m_{1}+\cdots+m_{k}$, follows by the Möbius Inversion Formula.

With the Witt identity can be associated the following diagram: a collection of $k$ directed planar loops $a_{1}, \ldots, a_{k}$ having only one vertex $P$ in common as, for example, in Figure 1. For later purposes it is required that no two loops have a common tangent vector at $P$. With each circular word $w$ of the kind considered is associated a closed circular path $p$ in a counterclockwise direction (in Figure 1).

${ }^{1}$ Research supported by a grant from the Michigan Institute of Science and Technology and NSF G 14093. 


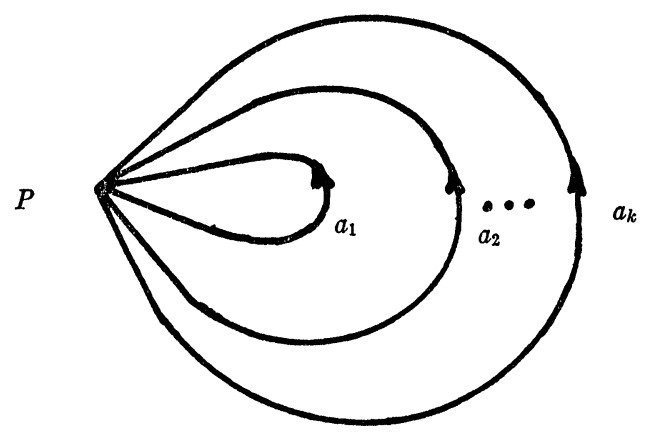

FIGURE 1

With each word one associates a monomial in $z_{1}, z_{2}, \cdots, z_{k}$ where the exponent of $z_{i}$ gives the number of times the loop $a_{i}$ is used. With the corresponding path the same monomial is associated. Then $M\left(m_{1}, \cdots, m_{k}\right)$ is the number of closed, circular, nonperiodic, counterclockwise (and therefore one-way) paths which use loop $a_{i}$ exactly $m_{i}$ times, $1 \leqq i \leqq k$.

Now let $b_{1}, b_{2}, \cdots, b_{k}$ generate a free group $X$. Consider only circular reduced words of the same length and period, where reduced means that $a_{i}$ and $a_{i}^{-1}$ never are adjacent in the circular order. Parenthetically, it should be observed that there is a 1-1 correspondence between complete conjugate classes in the free group $X$ and the circular reduced words. Let $n_{i}^{+}$be the number of occurrences of $b_{i}$ in the circular reduced word, $n_{\bar{i}}^{-}$be the number of occurrences of $b_{i}^{-1}$ in the word, and $n_{i}=n_{i}^{+}+n_{\bar{i}}^{-}, i=1,2, \cdots, k$.

If the diagram, such as in Figure 1, with $b$ 's instead of $a$ 's, is fixed, then with each circular reduced word with the same length and period one can associate a closed circular nonperiodic path on the loops with no "immediate reversals." This last means that $b_{i}$ and $b_{i}^{-1}$ are never adjacent. Note that the paths are no longer necessarily one-way. Now with each of the above paths consider the algebraic number of revolutions of the tangent vector. (It should be noted that the rotation of the tangent vector at a corner like $P$ is counted by taking that angle of rotation which is in absolute value less than $\pi$. For example if in the word $b_{k} b_{2}^{-1}$ appears then the induced rotation of the tangent vector at $P$ is given by the solid angle rather than the broken line angle in Figure 2.) Let $N\left(n_{1}, \cdots, n_{k} ; 1\right)$ be the number of these circular reduced words of the same length and period with parameters $n_{1}, n_{2}, \cdots, n_{k}$ and such that in the associated path the algebraic number of revolutions of the tangent vector is odd. Let $N\left(n_{1}, \cdots, n_{k} ;-1\right)$ be the number of analogous paths where the 


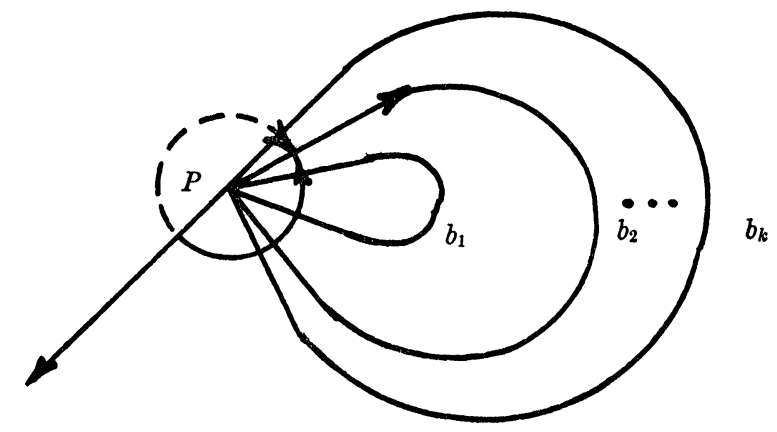

FIGURE 2

algebraic number of revolutions of the tangent vector is even. Let $z_{1}, \cdots, z_{k}$ be as before. Then a special case of Theorem 1 of [S, p. 205] yields the following analogue to (1):

(2)

$$
\begin{array}{r}
\prod_{1, \cdots, n_{k \geq 0}}\left(1+z_{1}^{n_{1}} \cdots z_{k}^{n_{k}}\right)^{N\left(n_{1}, \cdots, n_{k i} 1\right)}\left(1-z_{1}^{n_{1}} \cdots z_{k}^{n_{k}}\right)^{N\left(n_{1}, \cdots, n_{k} ;-1\right)} \\
=\left(\prod_{1 \leq j \leq k}\left(1+z_{j}\right)\right)^{2} .
\end{array}
$$

This comes from the special case of Theorem 1 where the planar graph consists of $n$ loops with a single common vertex, nontangential intersection at the vertex and such that of every pair of loops one is within the other. It should be noted that if another diagram such as in Figure 3 be considered, then for a given word the algebraic number of revolutions of the tangent vector depends on the diagram, i.e. whether it be as in Figure 1 or Figure 3 , but $N\left(n_{1}, \cdots, n_{k} ; 1\right)$ and $N\left(n_{1}, \cdots, n_{k} ;-1\right)$ are independent of the diagrams. Of course, the

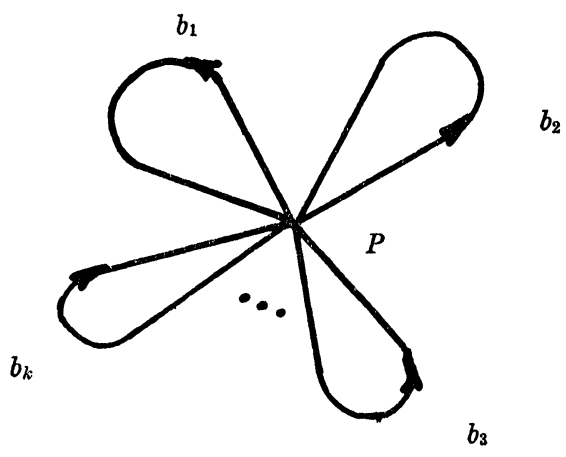

Figure 3 
basic remarkable feature about (2) is that a casual inspection of the formal product leads one to expect a formal multivariate power series with integral coefficients, but (2) indicates that the series breaks down to a polynomial with nonnegative integer coefficients. Theorem 1 of [S] is a generalization of (2) from diagrams like Figure 1 and Figure 3 to any planar 1-cycle with sufficient smoothness so that winding numbers can be defined. It constitutes a relation between the fundamental group and the first homology group over the integers mod 2 of this planar 1-cycle. An analogous relation between the two groups for 1-cycles in 3-space might very well crack the long attacked 3-dimensional Ising problem.

Gleason has pointed out that the weights +1 , and -1 which occur in (2) both as coefficients of the monomial and in the exponent of the binomial may be supplemented by a weight 0 which is applied to circular reduced words of different length and period. With this formulation (2) may be written with an obvious third factor so the the formal product could be taken over all circular reduced words and the exponents ignored. This gives the formula a more symmetric appearance. Moreover, the weight is a function taking values +1 , -1 , or 0 on complete conjugate classes of $X$ and so suggests the Möbius function. In the classical case, the domain of the Möbius function is the set of positive integers, but Delsarte has generalized this to where the domain is the set of finite abelian groups and where if the group is cyclic the new Möbius function assumes the same value that the classical Möbius function assumes for the order of the group. The author knows of no "Möbius function" theory with domain the complete conjugate classes of a free group on a finite number of generators.

However, E. P. Wigner in On representations of certain finite groups, American Journal of Mathematics (1941), p. 59 refers to a function of irreducible unitary representations of finite groups. This function is the sum of the characters of the squares of the group elements divided by the order of the group and has range in $\{1,-1,0\}$. Since for finite groups there is a one to one correspondence between characters of irreducible unitary representations and complete conjugate classes, this function looks like the weight of this note. However, no useful exploitation of this fact has been made.

A strictly algebraic and nongeometric proof of (2) would be in order when one considers the independence of the $N$ functions on the particular diagram. Such an algebraic proof might shed light on the Ising problem in three dimensions. It was in the hope of stimulating such light that this note was written. 


\section{BIBLIOGRAPHY}

H. M. Hall, The theory of groups, Macmillan, New York, 1959.

S. S. Sherman, Combinatorial aspects of the Ising model for ferromagnetism. I. A conjecture of Feynman on paths and graphs, J. Mathematical Phys. 1 (1960), 202-217.

W. E. Witt, Treue Darstellung Liescher Ringe, J. Reine Angew. Math. 177 (1937), 152-160.

Wayne State University 